\title{
Preliminary assessment of patient and physician satisfaction with the use of teleconsultation in urology during the COVID-19 pandemic
}

\author{
Ugo Pinar ${ }^{1}\left[\right.$. Julien Anract ${ }^{1}$. Ophélie Perrot ${ }^{1} \cdot$ Thomas Tabourin $^{1} \cdot$ Emmanuel Chartier-Kastler ${ }^{1}$. Jerome Parra ${ }^{1}$. \\ Christophe Vaessen ${ }^{1} \cdot$ Alexandre de La Taille $^{2} \cdot$ Morgan Roupret $^{1}$
}

Received: 8 May 2020 / Accepted: 30 August 2020 / Published online: 9 September 2020

(c) Springer-Verlag GmbH Germany, part of Springer Nature 2020

\begin{abstract}
Purpose Lockdown during the COVID-19 pandemic compelled urologists to change access to healthcare, especially for oncology patients. Teleconsultation is a safe way to receive medical advice without a risk of infection, and was implemented urgently in our academic centres. Our purpose was to evaluate patient and physician satisfaction with teleconsultation set up during the COVID-19 pandemic.

Methods From March 16th 2020, all face-to-face consultations were cancelled in France, except for emergencies. Teleconsultation was started immediately by five senior urologists in two academic hospitals. All patients received an email survey including the validated Teleconsultation Satisfaction Questionnaire (TSQ) and demographic questions. Data were collected prospectively. Physicians also responded to the TSQ. Patient satisfaction was measured objectively with the validated 14-item TSQ. Each item was scored on a 5-point Likert scale. Factors associated with positive satisfaction with teleconsultation were assessed by multivariable logistic regression.

Results Overall, 105 patients replied to the survey (91.3\%). Median age was 66 years (IQR: 55-71) and 95 were men (90.5\%). Median overall TSQ score was 67 (IQR: 60-69); teleconsultation was judged to be a good experience by 88 patients (83.8\%) and four physicians $(80 \%)$. Patients who met their surgeon for the first time were more likely to have a good experience $(\mathrm{OR}=1.2$ [95\% CI 1.1-1.5], $p=0.03)$.

Conclusion Introduced rapidly during the COVID-19 lockdown, urology teleconsultation attained a high level of satisfaction among both patients and physicians. A major change in telemedicine use is foreseen in the post COVID-19 era.
\end{abstract}

Keywords Telemedicine $\cdot$ Urology $\cdot$ Coronavirus $\cdot$ COVID-19

\section{Introduction}

The coronavirus disease 2019 (COVID-19) pandemic has led many countries to total lockdown because of the contagious nature of the virus and the lack of effective treatment. In France, lockdown was pronounced on March 17th, 2020, for at least 2 months. The whole healthcare system

Morgan Roupret

morgan.roupret@aphp.fr

1 Urology Department, Sorbonne University, GRC 5, Predictive Onco-Urology, Hôpital la Pitié-Salpêtrière, 75013 Paris, France

2 APHP, Henri Mondor Hôpital, Urology, Hôpitaux Universitaires Henri Mondor, 94010 Creteil, France has been massively impacted to accommodate COVID-19 patients, especially intensive care units. On March 16th, the French Association of Urology recommended that every non-urgent consultation should be delayed and non-urgent surgery postponed [1]. This major decision could have a negative impact on patients, particularly those diagnosed with urological cancers [2]. Patients could also neglect relevant symptoms placing them in a dangerous situation. An important question that arises is "How will we catch-up with all the cancelled or delayed consultations?" In this context, French urologists have urgently set up teleconsultation facilities to provide patients with the best possible care without compromising their safety.

Telemedicine consultation was first developed in the late 1960s, mainly in psychiatry, and consists of the delivery of healthcare services by healthcare professionals where 
distance is a critical factor [3, 4]. It is currently emerging as a way of providing medical services whilst respecting social distancing and reducing the spread of the virus within patient/physician populations. Improved outcomes, userfriendliness, low cost and decreased travel time are factors affecting telehealth effectiveness and efficiency $[5,6]$. For some physicians and patients, this new tool has rarely been evaluated, particularly in urology, and even less so during a worldwide pandemic. Our aim was to assess patient and physician satisfaction with teleconsultation used during the COVID-19 pandemic.

\section{Materials and methods}

\section{Study design}

This prospective, bi-centric study was carried out in two academic hospitals in Paris, France. Primary outcome was patient satisfaction with teleconsultation using the validated Telemedicine Satisfaction Questionnaire (TSQ) [7]. Secondary outcomes were predictive factors of adhesion to telemedicine and physician satisfaction.

\section{Telemedicine appointment}

All patients who were scheduled for a conventional consultation were given the opportunity to have a medical teleconsultation by the urology secretary. If they accepted, they were e-mailed detailed instructions. On the day and hour of the appointment, both physicians and patients could meet in a virtual room through the Doctolib $\odot$ website. Doctolib $\odot$ is a French company commonly used in France that manages medical appointments; it proposed teleconsultation as soon as the COVID-19 situation emerged. Patients and physicians could access the interface via a website (https://www. doctolib.fr), could speak to and see each other and exchange medical documents (laboratory reports, imaging reports, or prescription) through a secured encrypted platform.

\section{Patient population}

Patients who had a urological teleconsultation were systematically asked to answer the survey at the end of the clinical consultation. The teleconsultations were led by five senior urologists with more than 15 years' experience in the field in two academic hospitals in Paris (Hospital Henri-Mondor and Hospital Pitié-Salpêtrière) between March 30th and April 13th. Approved consent was obtained at the end of the consultation. Each patient was e-mailed a 20 -item questionnaire after their consultation and received a phone call in the case of further questions.

\section{Data collection}

The TSQ is a validated 14-item questionnaire developed in 2003 to evaluate patient satisfaction with telemedicine [7]. The questionnaire is composed of three main components: quality of care provided (TSQ1), similarity to faceto-face encounter (TSQ2) and perception of the interaction (TSQ3). The TSQ uses a 5-point Likert scale ranging from "Strongly disagree" (1) to "Strongly agree" (5). TSQ score varies from 14 to 70 , TSQ1 from 8 to 40 , TSQ2 from 5 to 25 and TSQ3 from 1 to 5 . Its content and construct have been validated for internal consistency reliability [8]. Total TSQ score $>56$ was considered a good experience for the patient.

In addition to the TSQ, various demographic questions were added to the survey. Patterns of use of smartphones and the internet were also assessed. All questionnaires were compiled in a unique survey that was sent to each patient who gave their consent.

\section{Statistical analysis}

Quantitative variables are described as median and interquartile range (IQR) and qualitative variables as number and percentage. For the TSQ, results are reported as a mean score and standard deviation (SD) for each item. The Chi-2 test and Wilcoxon test were performed to compare qualitative and quantitative variables, respectively. Logistic regression was performed to determine the predictive factors for a good experience. Statistical significance was set at $p<0.05$. All tests were 2 -sided. Analyses were performed using R version 3.6.2. (2009-2019 RStudio, Inc.).

\section{Results}

\section{Patient demographics}

Overall, 105 patients (95 men and 10 women) with a median age of 66 years (IQR: 55-71) responded to the online questionnaire, a response rate of $91.3 \%$ (Table 1). Most of the participants underwent their consultation for follow-up or oncological urology (Fig. 1). Of the participants, $10(9.5 \%)$ had already experienced a teleconsultation with another physician and 33 (31.4\%) met their urologist for the first time. Three patients $(2.9 \%)$ were unable to complete the consultation due to network problems and it was converted into a simple phone call. 
Table 1 Demographic characteristics of the study population $(N=105)$

\begin{tabular}{ll}
\hline Characteristic & Study population \\
\hline Age, years (median, (IQR)) & $66(55-71)$ \\
Gender, $n(\%)$ & \\
$\quad$ Female & $10(9.5)$ \\
$\quad$ Male & $95(90.5)$ \\
First teleconsultation, $n(\%)$ & $95(90.5)$ \\
Available material for teleconsultation, $n(\%)$ & $104(99)$ \\
Smartphone owner, $n(\%)$ & $100(95.2)$ \\
First consultation with this physician, $n(\%)$ & $33(31.4)$ \\
Possible isolation for teleconsultation, $n(\%)$ & $102(97.1)$ \\
Physician, $n(\%)$ & \\
1 & $15(14.3)$ \\
2 & $18(17.1)$ \\
3 & $18(17.1)$ \\
4 & $17(16.2)$ \\
5 & $37(35.2)$ \\
\hline
\end{tabular}

\section{Satisfaction with teleconsultation}

Teleconsultation was judged to be a "good experience" for 88 patients $(83.8 \%)$ and for four physicians $(80 \%)$ (Table 2). Ninety-four patients $(89.5 \%)$ considered that their medical issue was solved during the teleconsultation. The level of added stress was low with $<10 \%$ of responders concerned.

Overall TSQ score was 67 (IQR: 60-69), TSQ1 (quality of care provided) scored 38 (IQR: 33-40) while TSQ2 (similarity with face-to-face encounter) scored 24 (IQR: 22-25) (Table 3). Women had a significantly lower total TSQ score than men (61 (IQR: 56.5-65.5) vs. 67 (IQR:
60.5-69.5), respectively; $p=0.05)$. There was no significant difference of teleconsultation satisfaction among urological type of disease. Median TSQ [IQR] was 66.5 [62.3-69.8] for patients who had oncological motives versus 67 [59-69] for patients with non-oncological motives $(p=0.9), 63$ [58-68] for functional consultation versus 67 [60.3-70] for non-functional consultation $(p=0.1)$ and 68.5 [60-70] for general urology versus 66 [60-69] for non-general urological consultation $(p=0.3)$.

On multivariable logistic regression, patients who met their surgeon for the first time were more likely to have a good experience $(\mathrm{OR}=1.2$ [95\% CI 1.1-1.5], $p=0.03$ ). Gender, age, reason for consultation and previous teleconsultations were not associated with a good teleconsultation experience (Table 4).
Table 2 Patient and physician satisfaction with teleconsultation

\begin{tabular}{lll}
\hline & \multicolumn{1}{c}{ Patients $(N=105)$} & Physicians $(N=5)$ \\
\hline TSQ total, $n(\%)$ & & \\
$56-70$ & $88(83.8)$ & $4(80)$ \\
$42-56$ & $16(15.2)$ & $1(20)$ \\
$<42$ & $2(2)$ & 0 \\
Added stress because of teleconsultation, $n(\%)$ & \\
Yes & $10(9.5)$ & $0(0)$ \\
No & $95(90.5)$ & $5(100)$ \\
Medical issue solved with teleconsultation, $n(\%)$ & \\
Yes & $94(89.5)$ & NA \\
No & $11(10.5)$ & NA \\
\hline
\end{tabular}

\section{CONSULTATION DETAILS}

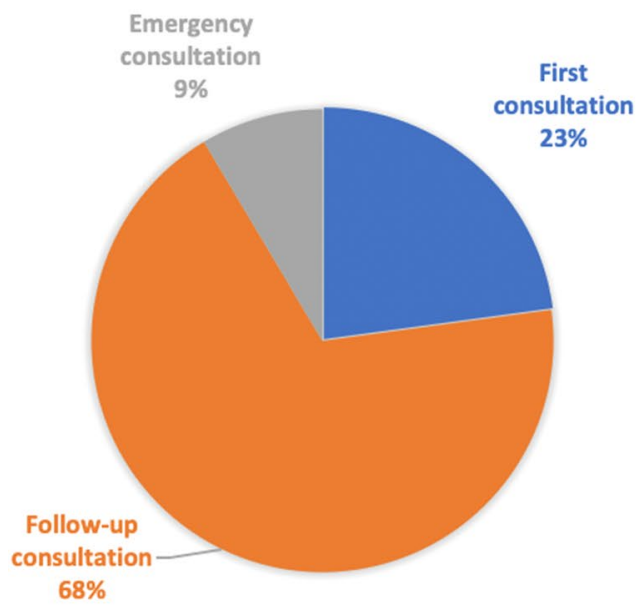

\section{CONSULTATION CAUSE}

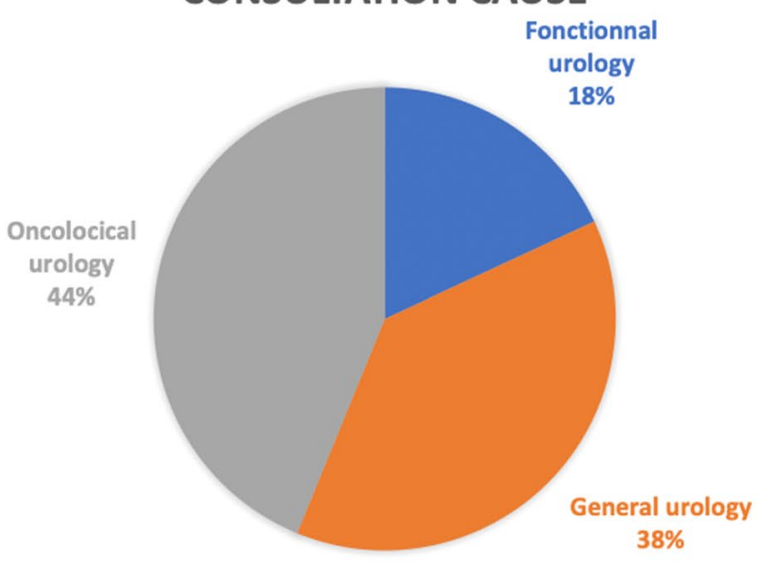

Fig. 1 Details and reasons for the teleconsultations. Most of the consultation led were for urological follow-up $(n=72,68 \%)$ in the field of oncology $(n=46,44 \%)$. Overall 24 patients $(23 \%)$ underwent their first consultation with their urologist 


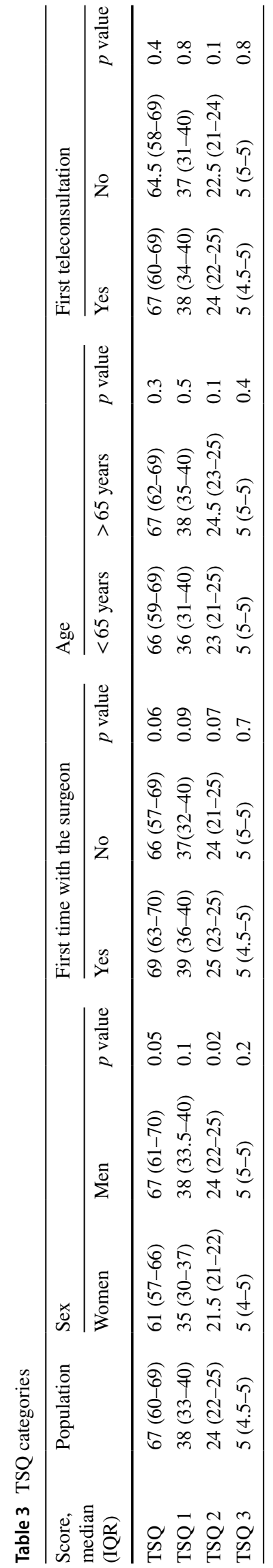

Table 4 Variables associated with greater satisfaction

\begin{tabular}{|c|c|c|c|c|}
\hline $\mathrm{TSQ}>56$ & $\begin{array}{l}\text { OR }(95 \% \text { CI }) \\
\text { Univariable }\end{array}$ & $p$ value & $\begin{array}{l}\text { OR }(95 \% \mathrm{CI}) \\
\text { Multivariable }\end{array}$ & $p$ value \\
\hline \multicolumn{5}{|l|}{ Gender } \\
\hline Male & 1 (Reference) & NA & 1 (Reference) & NA \\
\hline Female & $0.4(0.1-1.8)$ & 0.2 & $0.9(0.7-1.2)$ & 0.5 \\
\hline Age, years & $1(1.0-1.1)$ & 0.1 & $1(0.9-1.0)$ & 0.1 \\
\hline \multicolumn{5}{|c|}{ Teleconsultation experience } \\
\hline Not the first time & 1 (Reference) & NA & 1 (Reference) & NA \\
\hline $\begin{array}{l}\text { First teleconsul- } \\
\text { tation }\end{array}$ & $2.3(0.6-9.0)$ & 0.2 & $1.1(0.8-1.4)$ & 0.6 \\
\hline \multicolumn{5}{|c|}{ Consultation with physician } \\
\hline $\begin{array}{l}\text { Previous consul- } \\
\text { tation } \\
\text { First consultation }\end{array}$ & $\begin{array}{l}1 \text { (Reference) } \\
2.9(1.0-10.9)\end{array}$ & $\begin{array}{l}\text { NA } \\
0.07\end{array}$ & $\begin{array}{l}1 \text { (Reference) } \\
1.2(1.1-1.5)\end{array}$ & $\begin{array}{l}\text { NA } \\
0.03\end{array}$ \\
\hline \multicolumn{5}{|l|}{ Consultation motive } \\
\hline General urology & 1 (Reference) & NA & 1 (Reference) & NA \\
\hline Functional & $1.3(0.2-2.5)$ & 0.6 & $1(0.8-1.3)$ & 0.9 \\
\hline Oncology & $0.7(0.5-3.9)$ & 0.5 & $1.1(0.9-1.3)$ & 0.5 \\
\hline
\end{tabular}

\section{Discussion}

In this study, level of satisfaction with teleconsultation was high and was consistent with previous studies [5,9]. Several criteria associated with the patients' acceptance of teleconsultation were identified and must be considered in the long-term development of teleconsultation in urology. First, patients should embrace teleconsultation for its low cost and decreased travel time [10]. A high-quality service and improved access to care are also necessary conditions for teleconsultation sustainability [11]. Providers of teleconsultation should embrace it for its reduced waiting times, decreased number of readmissions and decreased number of missed appointments $[12,13]$.

In this study we evaluated patients' satisfaction with telemedicine in urology, used as a "plan B" tool faced with the restrictions of the COVID-19 pandemic. Its multi-centric and prospective design, including the use of a validated satisfaction survey, strengthen our results. Surprisingly, older patients ( $>65$-years) had a similar level of satisfaction to younger patients. In general, older patients do not embrace change and can become anxious due to their perceptions of teleconsultation. Our results indicate that older patients are able to embrace mobile technology and mobile health devices [14].

Various studies assessed patient and physicians satisfaction with of teleconsultation during "normal times", few are found in the field of urology. Wang et al. reported an $85 \%$ of mean overall satisfaction for dermatologic teleconsultation with no differences regarding age, gender or consultation motive [15]. Patient and physician's concordance regarding teleconsultation satisfaction has already been studied and is consistent with our results. Schubert 
et al. evaluated 110 patients and 10 psychiatrist's satisfaction regarding teleconsultation [16]. They were both highly satisfied with teleconsultation with a high level of concordance between patient and provider responses.

Our results regarding level of patient satisfaction in the field of urology are consistent with previously published studies. A prospective randomized study evaluated video visit consultation versus traditional office visit for patients who underwent radical prostatectomy [17]. Efficiency and satisfaction were equivalent between the two groups of patients, with a reduced cost for the group who had video visit consultations. Satisfaction was also high (95\% of cases evaluating teleconsultation "very good" to "excellent") in a study gathering 97 veterans of whom telemedicine was delivered to remote locations for urological motives [18].

One of the major obstacles to teleconsultation is access to a high-speed network. A few patients complained of poor-quality videos with low resolution and some even had to end the video consultation. Moreover, the system does not allow the exchange of imaging data such as computed tomography scans or magnetic resonance images. Thus, physicians cannot visualize these important examinations. The fifth-generation $(5 \mathrm{G})$ wireless network with a high data rate and low latency could be useful to overcome these limitations in the future $[19,20]$.

Teleconsultation in urology will never replace face-toface encounters. The wide-reaching and urgent introduction of teleconsultation in our practice will probably be redefined in the post COVID-19 era. Whether or not this electronic tool is more suitable for follow-up of clinical appointments rather than initial consultations is uncertain. Physical examination remains extremely important, particularly in the first post-operative follow-up consultation. Conversely, oncological follow-up of kidney, bladder or prostate cancer could benefit from teleconsultations alternating with face-to-face encounters.

The COVID-19 pandemic has imposed unprecedented measures because of the rapid spread of the virus and the lack of pre-existing scientific data [21]. During the pandemic, many "non-urgent" surgeries have been cancelled or delayed, including oncological surgery. Thus there is a risk of an oncological healthcare crisis [2]. Although some prostate cancer management can be delayed for a few weeks with a low risk of progression, it is not recommended for bladder or testicular cancer as it can impact outcome and survival [22]. Therefore, teleconsultation seems to be an acceptable tool for both oncological followup and screening [23].

One limitation of this study lies in its small population size, but the very high response rate of our total population reduces this bias.

\section{Conclusion}

Teleconsultation in urology was an acceptable method to provide patients with safe access to healthcare during the COVID-19 pandemic. This tool has a high level of satisfaction for both patients and their physicians and could be developed in the future.

Author contributions UP: Participated in research design, data collection, writing of the paper, performance of the research, data analysis. JA: Participated in research design, writing of the paper, performance of the research, data analysis. OP: Participated in research design and data analysis. TT: Participated in research design and data analysis. EC-K: Participated in research design, writing of the paper and data collection. JP: Participated in writing of the paper and data collection. CV: Participated in writing of the paper and data collection. AT: Participated in research design, writing of the paper and data collection. MR: Participated in research design, writing of the paper, performance of the research, data analysis.

Funding The authors declare no funding. This research did not receive any specific Grant from funding agencies in the public, commercial, or not-for-profit sectors.

Availability of data and material Not applicable.

Code Availability Not applicable.

\section{Compliance with ethical standards}

Conflict of interest The authors declare no conflict of interest.

Informed consent For this study oral consent was obtained for every patient.

\section{References}

1. Association Française d'urologie (2020) Informations coronavirus (COVID-19). https://www.urofrance.org/base-bibliographique /informations-coronavirus-covid-19. Accessed 9 Apr 2020

2. Oderda M, Marra G, Roupret M et al (2020) The impact of Covid19 outbreak on uro-oncological practice across Europe: which burden of activity are we facing ahead? Press EAU Eur Urol Covid Resour Cent 78:124-126

3. Dwyer TF (1973) Telepsychiatry: psychiatric consultation by interactive television. Am J Psychiatry 130:865-869. https://doi. org/10.1176/ajp.130.8.865

4. Benschoter RA, Eaton MT, Smith P (1965) Use of videotape to provide individual instruction in techniques of psychotherapy. J Med Educ 40:1159-1161. https://doi.org/10.1097/00001888196512000-00006

5. Kruse CS, Krowski N, Rodriguez B et al (2017) Telehealth and patient satisfaction: a systematic review and narrative analysis. BMJ Open. https://doi.org/10.1136/bmjopen-2017-016242

6. de la Torre-Díez I, López-Coronado M, Vaca C et al (2015) Costutility and cost-effectiveness studies of telemedicine, electronic, and mobile health systems in the literature: a systematic review. Telemed J E Health Off J Am Telemed Assoc 21:81-85. https:// doi.org/10.1089/tmj.2014.0053 
7. Yip MP, Chang AM, Chan J, MacKenzie AE (2003) Development of the Telemedicine Satisfaction Questionnaire to evaluate patient satisfaction with telemedicine: a preliminary study. J Telemed Telecare 9:46-50. https://doi.org/10.1258/135763303321159693

8. Langbecker D, Caffery LJ, Gillespie N, Smith AC (2017) Using survey methods in telehealth research: a practical guide. J Telemed Telecare 23:770-779. https://doi.org/10.1177/1357633X17 721814

9. Georgsson M, Staggers N (2016) Quantifying usability: an evaluation of a diabetes mHealth system on effectiveness, efficiency, and satisfaction metrics with associated user characteristics. J Am Med Inform Assoc JAMIA 23:5-11. https://doi.org/10.1093/jamia locv099

10. Müller KI, Alstadhaug KB, Bekkelund SI (2016) Acceptability, feasibility, and cost of telemedicine for nonacute headaches: a randomized study comparing video and traditional consultations. J Med Internet Res 18:e140. https://doi.org/10.2196/jmir.5221

11. Gibson KL, Coulson H, Miles R et al (2011) Conversations on telemental health: listening to remote and rural First Nations communities. Rural Remote Health 11:1656

12. Breen P, Murphy K, Browne G et al (2010) Formative evaluation of a telemedicine model for delivering clinical neurophysiology services part I: utility, technical performance and service provider perspective. BMC Med Inform Decis Mak 10:48. https:// doi.org/10.1186/1472-6947-10-48

13. Iqbal A, Raza A, Huang E et al (2017) Cost effectiveness of a novel attempt to reduce readmission after ileostomy creation. JSLS. https://doi.org/10.4293/JSLS.2016.00082

14. Kruse CS, Mileski M, Moreno J (2017) Mobile health solutions for the aging population: a systematic narrative analysis. J Telemed Telecare 23:439-451. https://doi.org/10.1177/1357633X16 649790

15. Wang Y-C, Ganzorig B, Wu C-C et al (2018) Patient satisfaction with dermatology teleconsultation by using MedX. Comput
Methods Progr Biomed 167:37-42. https://doi.org/10.1016/j. cmpb.2018.10.015

16. Schubert NJ, Backman PJ, Bhatla R, Corace KM (2019) Telepsychiatry and patient-provider concordance. Can J Rural Med Off J Soc Rural Phys Can J Can Med Rurale J Off Soc Med Rurale Can 24:75-82. https://doi.org/10.4103/CJRM.CJRM_9_18

17. Viers BR, Lightner DJ, Rivera ME et al (2015) Efficiency, satisfaction, and costs for remote video visits following radical prostatectomy: a randomized controlled trial. Eur Urol 68:729-735. https ://doi.org/10.1016/j.eururo.2015.04.002

18. Chu S, Boxer R, Madison P et al (2015) Veterans affairs telemedicine: bringing urologic care to remote clinics. Urology 86:255260. https://doi.org/10.1016/j.urology.2015.04.038

19. Tian W (2020) Exploration and prospect of $5 \mathrm{G}$ application in telemedicine. Zhonghua Wai Ke Za Zhi 58:1-4. https://doi. org/10.3760/cma.j.issn.0529-5815.2020.01.001

20. Lacy AM, Bravo R, Otero-Piñeiro AM et al (2019) 5G-assisted telementored surgery. Br J Surg 106:1576-1579. https://doi. org/10.1002/bjs. 11364

21. Shimizu K (2020) 2019-nCoV, fake news, and racism. Lancet 395:685-686. https://doi.org/10.1016/S0140-6736(20)30357-3

22. Russell B, Liedberg F, Khan MS et al (2020) A systematic review and meta-analysis of delay in radical cystectomy and the effect on survival in bladder cancer patients. Eur Urol Oncol 3:239-249. https://doi.org/10.1016/j.euo.2019.09.008

23. Connor MJ, Winkler M, Miah S (2020) COVID-19 pandemic-is Virtual Urology Clinic the answer to keeping the cancer pathway moving? BJU Int. https://doi.org/10.1111/bju.15061

Publisher's Note Springer Nature remains neutral with regard to jurisdictional claims in published maps and institutional affiliations. 